Supporting Information

\title{
Probing in Space and Time the Nuclear Motion Driven by Nonequilibrium Electronic Dynamics in Ultrafast Pumped $\mathrm{N}_{2}$
}

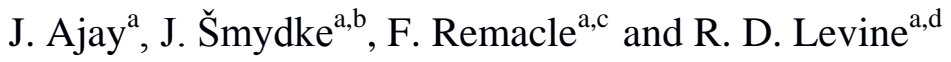

a. The Fritz Haber Center for Molecular Dynamics and Institute of Chemistry, The Hebrew University of Jerusalem, Jerusalem 91904, Israel

b. Department of Radiation and Chemical Physics, Institute of Physics, Academy of Sciences of the Czech Republic, 18221 Praha 8, Czech Republic.

c. Département de Chimie, B6c, Université de Liège, B4000 Liège, Belgium

d. Crump Institute for Molecular Imaging and Department of Molecular and Medical Pharmacology, David Geffen School of Medicine and Department of Chemistry and Biochemistry, University of California, Los Angeles, CA 90095

- A movie of the $\Sigma$ and $\Pi$ state dynamics during and after the pump pulse (cf. Figure 2 a,b,c)

- $\quad$ Figure S1a,b: $\Pi$ and $\Sigma$ dipole plotted up to $180 \mathrm{fs}$ (cf. Figure 3)

- Figure S2: Action of the probe on the $\Sigma$ state (cf. Figure 5) 
Figure S1a,b: $\Pi$ and $\Sigma$ dipole plotted up to $180 \mathrm{fs}$ (cf. Figure 3)
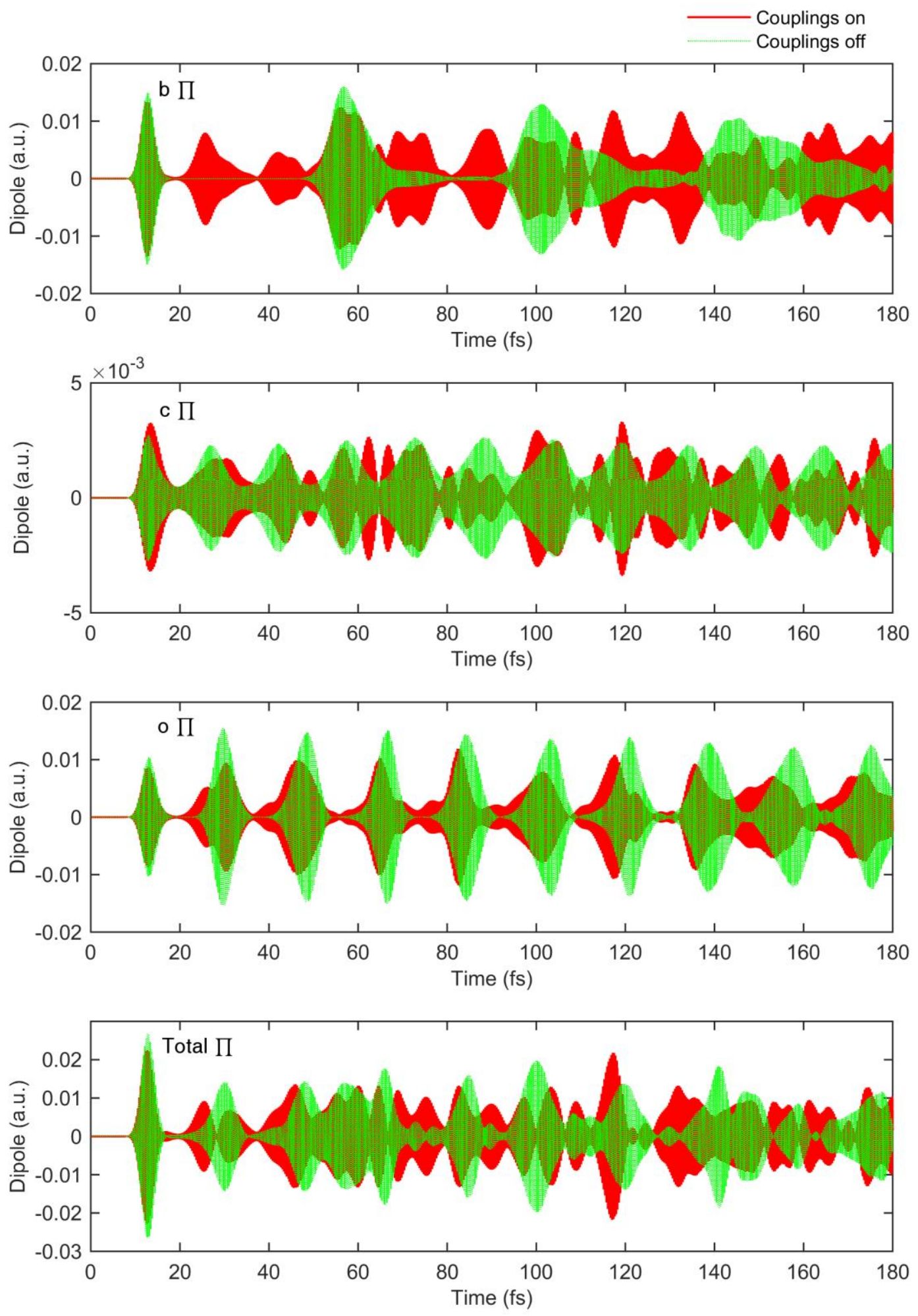

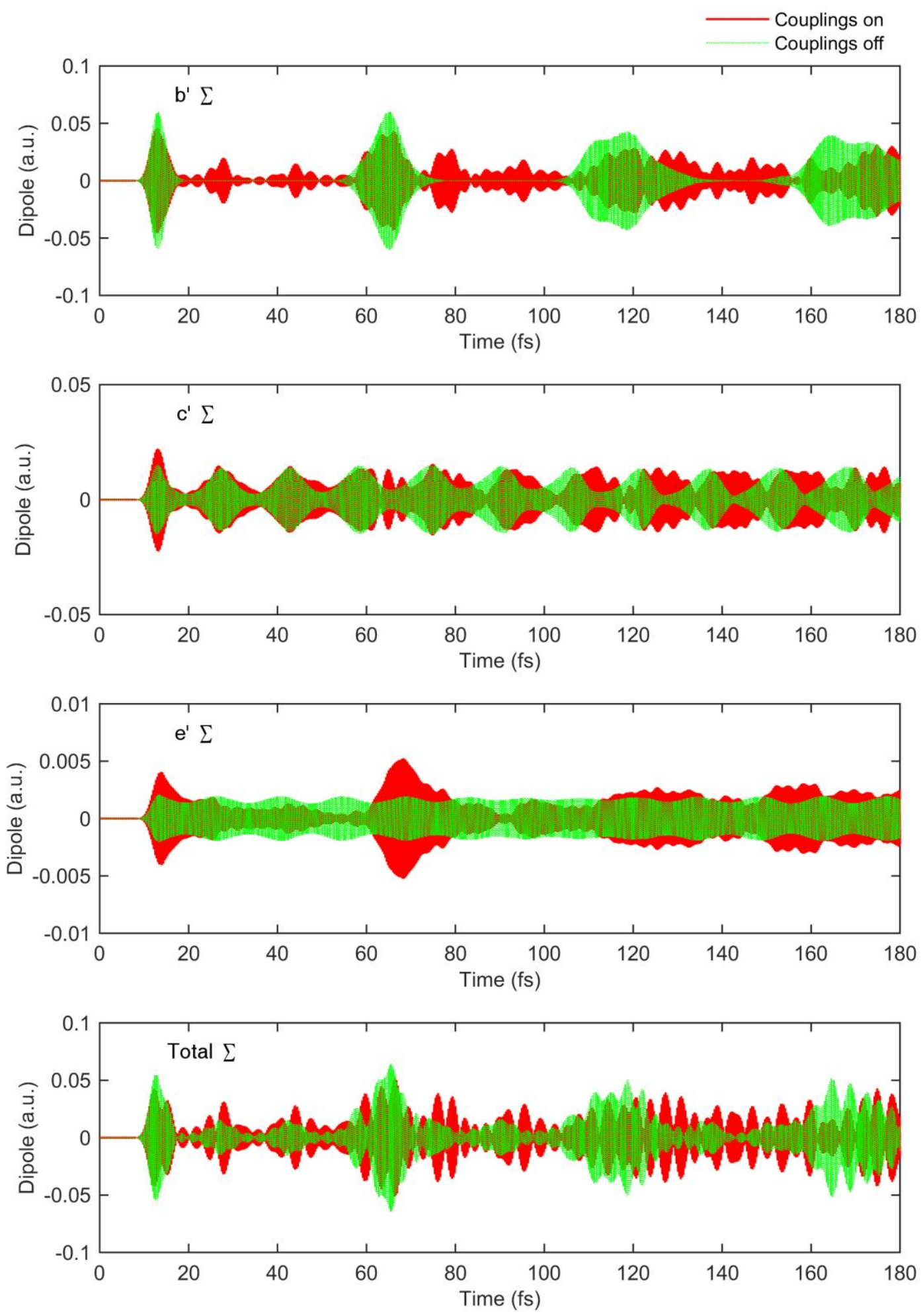
Figure S2: Action of the probe on the $\Sigma$ state (cf. Figure 5)
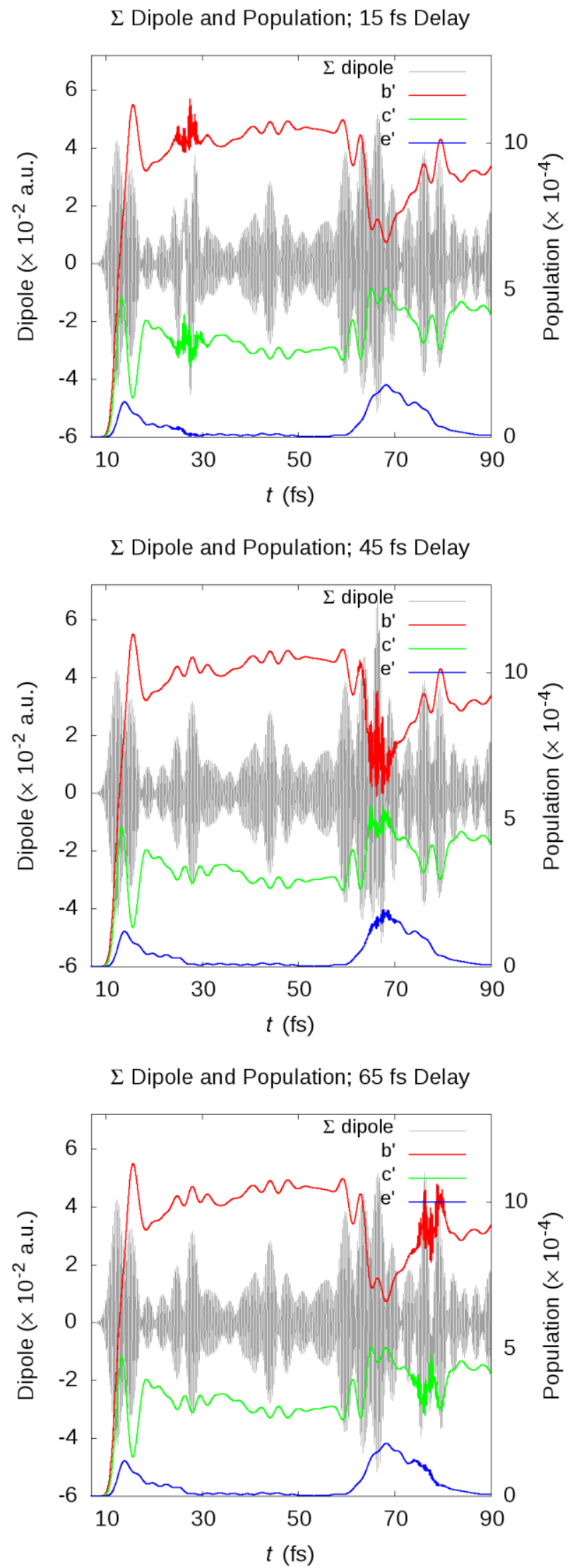